\title{
Non-Contractual Trust, Design, and Human and Computer Interactions
}

\author{
Elisabeth Davenport (Organizer) \\ Napier University Business School \\ Sighthill Court \\ Edinburgh EH11 4BN UK \\ +441314553337 \\ e.davenport@napier.ac.uk
}

\author{
Panelists and Affiliations \\ Mark Dibben, University of Aberdeen, cms055@abdn.ac.uk \\ Batya Friedman, University of Washington, batya@u.washington.edu \\ Steven Marsh, National Research Council of Canada, Steve.Marsh@iit.nrc.ca \\ Howard Rosenbaum, Indiana University, hrosenba@indiana.edu \\ Harold Thimbleby, Middlesex University, h.thinbleby@mdx.ac.uk
}

\begin{abstract}
How might trust be a component of human-computer based interaction? There are a number of dimensions involving different combinations of humans, systems and computer agents. Recent studies of trust in the workplace indicate that trust has many attributes, and that representations of trust in the virtual workplace must take account of differences in emphasis. [1] The panel members will work with a number of 'real world' scenarios that illustrate different aspects pertinent to trust and the morality of interactions
\end{abstract}

\section{Keywords}

Trust, design, interaction design, human values

\section{INTRODUCTION}

Though trust has been the topic of at least two recent $\mathrm{HCI}$ panels $[2,3]$ there is little consensus on how it may work or be constructed in the wired world. Congenial interactive systems must support a number of interactive relationships: humans trusting humans in contexts where

(C) Copyright on this material is held by they do not see each other; humans trusting systems; humans trusting agents who operate within systems; and agents trusting other agents. The panel will focus on situational trust, specifically the judgments made in social interactions, supported to a greater or lesser extent by computers. The panelists will address the problem from a number of different angles: as consultants, system builders, and facilitators. They will work with a number of scenarios at a micro level: first with a scenario from the world of commercial transactions; second, with a scenario based on Prisoner's Dilemma/game theory; third, a scenario drawn from fieldwork on user support for ecommerce.

Previous panels indicate that the topic provokes strong and conflicting audience response. Some consider trust to be an intuitive quality, a feeling that cannot be atomised, de-constructed and re-constructed. Others question the appropriateness of simple algorithms when the 'locus of trust' is likely to be diverse in any given situation. Others are suspicious of machines that behave as if they were to be trusted, as trust is a quality that is closely linked to human responsibilities. The panel may clarify what may or may not be said about trust in a given situation and identify areas that may be explored in other fora. 
FORMAT OF THE PANEL

Each of the members of the audience will be briefed with a page summary of 'threads' that will also indicate points that have provoked dissent or controversy in previous discussions of these positions. The panelists will be asked to make short position statements (seven minutes) in terms of the following 'threads':

1. the analysis of trust in a number of different interpersonal interactive contexts (Dibben)

2. representations of trust, by means of analytic formalisms, which may underly the conduct of computer agents (Marsh)

3. consumer trust in e-commerce vendors and services (Rosenbaum)

4. human values and perceptions of responsibility and distributive justice in $\mathrm{CHI}$ (Friedman and Thimbleby)

After a short panelist discussion to give each member a chance to comment on the stances of others, the audience will then be asked to direct questions to individual panelists (and threads), moderated by the panel coordinator, who will attempt to draw the threads together at the close of the panel session. The panel should have broad appeal, as the issues are pertinent to all of those who engage with agents and services in the context of human-computer interaction. It may be of particular interest to interaction designers.

\section{PANELIST DETAILS}

Mark Dibben lectures in the University of Aberdeen Management Studies Department, and works with a leading Scottish management consultancy applying models of trust and cooperation within a number of major Scottish companies. [4] $\mathrm{He}$ has identified three problematics in this respect. First, conceptualising and operationalising the nature and extent of an interaction or situation in which trust operates. Second, conceiving of a means to accurately represent the process of trust development in and between computer and human agents in and across situations. Third, attempting to measure a quality in development given an 'atomistic' or elemental approach to the subject that (probably) relies on quantity as its primary measuring mechanism.

Batya Friedman is an Associate Professor in the School of Computing at the University of Washington. She takes the position that an important component of trust in $\mathrm{CHI}$ is the design of technology that is responsive, or sensitive to, human values. Network security is a case in point: though much effort is directed toward developing the technical dimensions, little systematic work has investigated and integrated the corresponding dimensions of human values and user experience, and methodologies for this must be explored.
Steve Marsh is a researcher in the National Research Council of Canada. He is responsible for groundbreaking work on the formalisation of trust as a computational concept for use in Artificial Intelligence. [5] As an example of a trusting agent scenario, he will develop a prisoner's dilemma scenario based on his work as a builder of agent based trust systems. We have to be able to trust the agent to do for us what we want, and to feel that at any time we can regain control of it actions. How can we achieve this? One way is to embed social knowledge in the interface agent so as to enable it to facilitate trust between the user and itself.

Howard Rosenbaum is Associate Professor in the School of Library and Information Science, Indiana University, and a Fellow of the Centre for Social Informatics there. His current work focuses on the socio-technical contexts of electronic commerce. In this work, he is examining the components of the consumer's e-commerce experience which build trust in the firm.

Harold Thimbleby is Professor of Computing Research, Middlesex University. $\mathrm{He}$ is a member of IFIP 9.2.2. (Computers and Ethics), and his concerns with trust in user interfaces have resulted in papers on cryptography, artificial life, and on Trojan and computer viruses. He recently presented a paper on 'Justice in Computing' which puts the onus of trust on human, not computer agents. [6]

\section{REFERENCES}

1.Kramer, R.K. and T.R. Tyler (eds.) Trust in organizations. London: Sage, 1996

2.Friedman, B. and Thomas, J.C. Trust me, I'm accountable: trust and accountability online. Extended panel abstract. CHI 99 Extended Abstracts, New York: ACM, 1999, 79 - 80.

3.Davenport, E. et al. 'Artificial morality': representations of trust in interactive systems. In S. Brewster et al. (Editors). Human-Computer Interaction - INTERACT '99. Vol. II. Swindon: BCS:IFIP, 1999, 281-286

4.Dibben, M.R. (2000) Exploring Interpersonal Trust in the Entrepreneurial Venture. London: MacMillan.

5.Marsh, S. Trust in distributed artificial intelligence. In Castelfranchi and Werner, Artificial Social Systems, Springer LNAI, 330, 1996, 95 - 112.

6.Duquenoy, P. and Thimbleby, H. Justice and design. In A.M. Sasse and C. Johnson (Editors). Human-Computer Interaction - INTERACT'99. Vol. I. Amsterdam: IOS:IFIP, 1999, 281-286 